\title{
Hadronization revisited: the dynamics behind hadro-chemical equilibrium
}

\section{R. Stock*, University of Frankfurt}

The multiplicities of hadronic species, from pions to omega hyperons created both in elementary, and in highly relativistic nucleus-nucleus collisions, are known to exhibit a pattern that is very well reproduced within the framework of the statistical model - as applied, in its canonical form, to the former class of reactions, and in the grand canonical version to the latter. To understand the origin(s) of this apparent equilibrium we revisit, first, the hadronization models developed for $e^{+} e^{-}$annihilation to hadrons via partonic jet fragmentation that lead to a color pre-confinement state, occuring at the end of the perturbative QCD DGLAP evolution, which lends itself to color singlet formation that is then recast in terms of massive singlet clusters, in a non perturbative model. These clusters decay into the known hadron/resonance spectrum, the eventual hadronic species yield distribution thus being seen as the result of a perturbative evolution toward potential color singlet "mass" distributions that are subsequently acquiring excited non perturbative hadronic condensate structures, that finally decay statistically (under phase space governance) to on-shell hadrons/resonances. Thus the success of a classical canonical ensemble description is understood as the consequence of various stochastic elements occuring in the dynamical evolution that ends with hadronic freeze-out. Turning to hadronization in relativistic nucleus-nucleus collisions we take advantage of the above singlet cluster pre-hadronization picture. These clusters are spatially isolated objects in the jet hadronization evolution, but with given extended spatial size, of the order of $1 \mathrm{fm}$. Lacking a detailed model of partonic transport evolution in highly relativistic $\mathrm{A}+\mathrm{A}$ collisions we assume that hadronization occurs from similar color singlet clusters that will, however, overlap spatially owing to the extreme overall energy density. An ensuing cluster overlap coherence may be symbolically understood by means of a percolation model. Cluster overlap increases with $\sqrt{s}$, A and collision centrality. In the limit of an extended coherent volume, hadronization is free of local quantum number constraints. After super-cluster decay the yield distribution is captured by the grand canonical version of the statistical equilibrium model which features strangeness saturation (large volume limit).

Critical Point and Onset of Deconfinement

July 3-6 2006

Florence, Italy

\footnotetext{
* Speaker.
} 


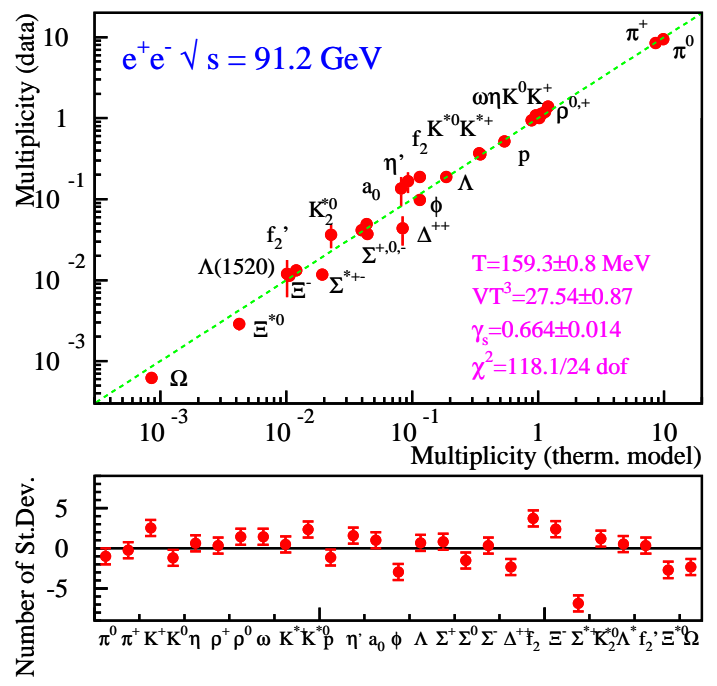

Figure 1: Hadron production in $e^{+} e^{-}$annihilation at $\sqrt{s}=91.2 \mathrm{GeV}$, with canonical model [6] fit.

\section{Introduction}

The average multiplicity of hadronic species made up by the three lightest quark flavours has been well characterized in a multitude of elementary collision processes, e.g. $e^{+} e^{-}, p p$ and $p \bar{p}$, and also in relativistic nucleus-nucleus collisions. In the present article we will be first concerned with LEP $e^{+} e^{-} \rightarrow$ hadron data at $\mathrm{Z}_{0}$ energy $(\sqrt{s}=92 \mathrm{GeV})$. The ideas about hadron production dynamics in such collisions center on a partonic shower evolution governed by perturbative QCD which ends in color singlet formation [1], in the form of nonperturbative QCD clusters [2,3] or strings [4], which finally decay to real hadrons and resonances $[3,5]$. The most remarkable property of the resulting multiplicity distributions, from pions to omega hyperons, is that they are well described by a classical canonical ensemble [6,7], i.e. by a state of statistical equilibrium.

Fig.1 illustrates the success of the statistical model description [6] for $\operatorname{LEP} e^{+} e^{-}$annihilation to hadrons. This is a striking outcome, considering the high energy that is initially distributed over a small number of partons. Ever since Hagedorns first systematic introduction of the statistical hadronization model [8] there has been fierce debate about how this apparent equilibrium feature could be either dynamically acquired ("thermalization") or, alternatively, be a feature of quantum mechanical decay of strings or clusters, occuring under dominance of the phase space weights corresponding to the QCD hadronic/resonance mass (spin etc.) spectrum. I.e. is the canonical ordering of multiplicities essentially an "acquired" or a "lost" property? The answer must be found mostly in the non perturbative phase (recall, however, the "pre-confinement" idea of Amati and Veneziano [1,9]), a task that might become tractable given the recent progress of npQCD hadron theory [10].

A remark of warning is in order here. The apparent canonical multiplicity order has often inspired the statement that the final hadronic state has "maximum entropy", in general. This is an inaccurate statement as is obvious e.g. from LEP di-jet final states which feature almost the opposite, near minimal entropy as far as the pencil-sharp track topology signature is concerned. 


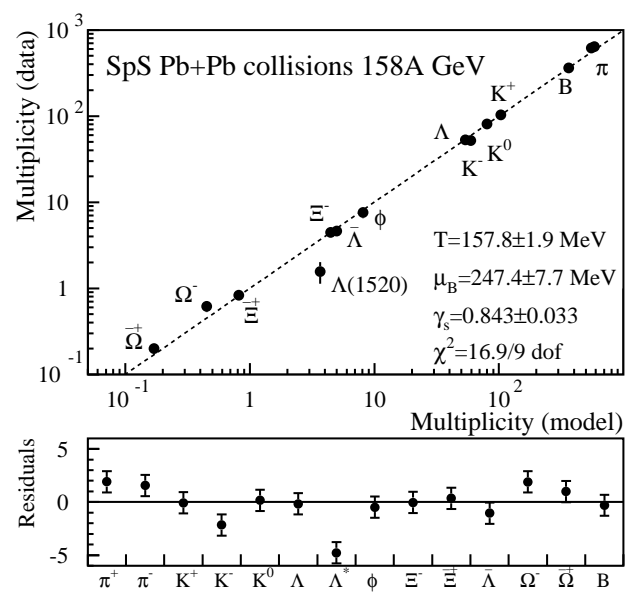

Figure 2: Hadron production measured in $4 \pi$ by NA49 in central $\mathrm{Pb}+\mathrm{Pb}$ collisions at $\sqrt{s}=17.3 \mathrm{GeV}$, compared to prediction [11] by the grand canonical model.

We revisit hadronization in elementary collisions for the following reason. It has turned out that the hadronization output from relativistic nucleus-nucleus collisions, as studied at the AGS, SPS and at RHIC, follows an analogous equilibrium ensemble pattern, this time represented by the grand canonical (GC) statistical ensemble [11, 12, 13, 14, 15]. A typical example [11] of a GC fit to the hadronic multiplicities, from pions to omega hyperons as observed by the SPS experiment NA49 in central $\mathrm{Pb}+\mathrm{Pb}$ collisions at $\sqrt{s}=17.3 \mathrm{GeV}$ is shown in Fig.2. The conceivable causes of equilibrium are more diverse here. The observed thermal equilibrium could be

a. dynamical achieved by partonic thermalization prior to hadronization $[16,17]$,

b. or be the outcome of stochastic and phase space effects governing the decay process to hadrons/resonances [18, 19, 20], during hadronization,

c. or, finally, be the effect of hadron/resonance inelastic rescattering cascades occuring in the dense medium right after hadronization $[21,22]$ such that hadronic freeze-out does not occur at, but after hadronization.

Note that, in option $\mathrm{c}$, the observed grand canonical temperature $(\mathrm{T} \approx 160 \mathrm{MeV}$ at top SPS and RHIC energy) should be only a lower limit to the hadronization transition temperature. The latter being an essential ingredient of the long-sought phase diagram of QCD matter, and the clarification of the dynamical origin of the grand canonical multiplicity pattern thus of crucial importance concerning the significance of nuclear collision data. Option a is of acute interest toward an explanation of the "elliptic flow" spatial emission pattern exhibited by the recent RHIC data [17] which appears to result from global hydrodynamic parton flow anisotropies created in the early partonic phase of the A+A dynamical evolution. Clearly, only option b describes the elementary collisions which should thus be revisited.

The idea of this paper is thus to try out the following line of argument: 
1. Revisit the pQCD analysis of the truly elementary process $e^{+} e^{-} \rightarrow$ di-jets $\rightarrow$ hadrons at LEP energy, in particular the transition phase at the end of the perturbative evolution leading to color singlet formation [1], a dynamical $\mathrm{pQCD}$ process that gives rise to "massive" singlet clusters $[2,3]$ which are recast into npQCD language "pre-hadronic" clusters. Interpreting them as a superposition of highly excited hadronic resonance states their subsequent quantum mechanical decay "gives birth" $[5,8,18,19,20]$ to the canonical ensemble population that is attested by the successful description within the modern version [6] of the Hagedorn model. This model also provides for a description of hadronization in $\mathrm{p}+\mathrm{p}$ collisions at SPS energy [7].

2. The latter observation suggests that the dynamical details of the partonic pQCD phase are washed out during the subsequent npQCD singlet cluster to on-shell hadron/resonance evolution. We note that this is only true for the multiplicity distribution of hadronic species composed of the two light quark species. The strangeness abundance appears to preserve memory of its initial suppression, requiring a fudge factor.

3. This cluster hadronization model might, thus, be universal. Its postulated equivalence to the hadronization scheme exploited in the string model $[4,5]$ is, thus, reassuring but falls beyond the scope of the present analysis. The model should thus be also applicable to hadronization in highly relativistic A+A collisions, e.g. from top SPS to RHIC energy, $17 \leq \sqrt{s} \leq=200$ $\mathrm{GeV}$, where the assumption of a partonic primordial dynamics appears equally plausible.

4. Such central collisions of $A \approx 200$ nuclei provide for a primordial reaction volume which is both extended far beyond typical confinement and hadronization cluster size (both of order $1 \mathrm{fm}$ ), and featuring a very high average energy density, well above the critical density of about $1 \mathrm{GeV} / \mathrm{fm}^{3}$ at which lattice QCD predicts the onset of deconfinement [23]. The primordial dynamics may thus establish a parton plasma state, as is indicated e.g. by RHIC observations of jet quenching [24] and elliptic flow formation [17]. Anyway the hadronic multiplicity distribution is universally well described, again, by the statistical hadronization model $[11,12,13,14,15]$ but now in its grand canonical form.

5. Finally: supposing that the hadronization process is essentially insensitive to the details of the preceding partonic evolution (point 2 above), the degree of partonic equilibration in a QGP might be of minor relevance to the hadronization outcome (option a above). However the high energy density, occuring in an extended volume, may change the singlet cluster decay mode, from one that occurs isolated in vacuum, in elementary collisions, to the decay of more extended complexes of overlapping clusters which decay in a quantum mechanically coherent manner. Formation of such "super-clusters" would be naively expected to increase with $\sqrt{s}$, nuclear projectile mass A, and collisions centrality, in accord with the observation that the transition from canonical to grand canonical hadronization indeed occurs smoothly, within these variables $[15,25,26]$. The transition from small system size at hadronization (elementary collisions), to large coherent system size (A+A collisions) - with a decay to the final hadrons/resonances occuring from spatially extended "super-clusters" - then appears to be reflected in the concurrent transition from a canonical to a grand canonical description. 
Note that it has been shown [15] that, in fact, the latter ensemble can be regarded as the large coherent volume limit of the former, as far as production of hadrons composed of the 3 light flavours is concerned.

It follows that our above option c, the hypothesis of equilibration through inelastic hadronic final state cascades occuring after hadronization [21, 22], is not required as the principally novel mechanism governing A+A hadronic multiplicity equilibrium - at least at very high $\sqrt{s}$ where hadrons should form directly from a preceding partonic evolution. We note, however, that anti-protons and anti-lambda hyperons may be subject to some final state annihilation, not as much at top RHIC energy where $\mu_{B} \rightarrow 0$ as at top SPS energy where the final hadronic cascade expansion is governed by a considerable net-baryon excess. Furthermore, the eventually observed $\Phi$ multiplicity may deviate from its primordial hadronization level, due to "coalescence" interaction with the high $\mathrm{K}^{+}+\mathrm{K}^{-}$density prevailing during the early cascade stage.

We also note that, toward the lower SPS and AGS energy domain, the above overall model of hadronic freeze-out directly from a preceding partonic phase becomes inapplicable as the freezeout temperatures obtained from grand canonical ensemble analysis $[11,12]$ fall well below of the parton-hadron coexistence line in the $\left[\mathrm{T}, \mu_{B}\right]$-plane that has recently been established by an extension of lattice QCD predictions to finite baryochemical potential [27]. This observation suggests the occurence of a finite interval in expansion time of the fireball, spent between hadronization and hadronic species freeze-out. This phase should indeed be subject to the inelastic rescattering cascades considered in rfs. [21, 22], our option c above.

In spite of such caveats I will concentrate here mainly on the question of what can be learned for A+A collisions, by revisiting the 1980s QCD jet hadronization models, developed e.g. for $e^{+} e^{-}$ annihilation $[1,2,3,4,5]$.

\section{Hadronization in $e^{+} e^{-}$annihilation}

What follows is a brief sketch of QCD models developed in the 1980s period where first comprehensive data concerning hadron formation in the, perhaps, most "elementary" QCD process of $e^{+} e^{-}$annihilation to jets of hadrons became available. At typical LEP collider energies, $\sqrt{s} \approx$ $100 \mathrm{GeV}$, this process is reflected, in the end, by a back-to-back di-jet configuration featuring about 20 hadrons. Its first step, $e^{+} e^{-}$annihilation, creates a virtual photon, labeled "virtual" as it contains the total initial c.m. energy of the $e^{+} e^{-}$pair but has zero momentum in that frame. In view of the energy law of real particles, $E^{2}=p^{2}+m^{2}$, this situation with $\mathrm{p}^{2}=0$ suggests the interpretation that a state of "virtual mass" $\mathrm{M}^{2}=\mathrm{E}_{c m}^{2}$ has been created. According to quantum mechanics its live-time is $\tau=1 / \mathrm{M}$ within which it decays to a quark - anti-quark pair. Next, over a period characterized by time scales $t$ such that $1 / M<t<t_{0}$, the primary partons develop into multi-parton cascades or showers by multiple gluon bremsstrahlung, as illustrated [1] in Fig.3.

These cascades, which tend to develop along the directions of the primary partons owing to the "collinear enhancement" in QCD matrix elements, are the precursors of the jets that are observed experimentally. This rapid, hard process is described by the so-called DGLAP-evolution of perturbative QCD; it leads from initial high virtuality (and large momentum transfer-squared $\mathrm{Q}^{2}$ ) to partons of "virtual mass" $1 / t_{0}$ where $t_{0}$ approaches the so-called QCD cutoff scale. It is defined 


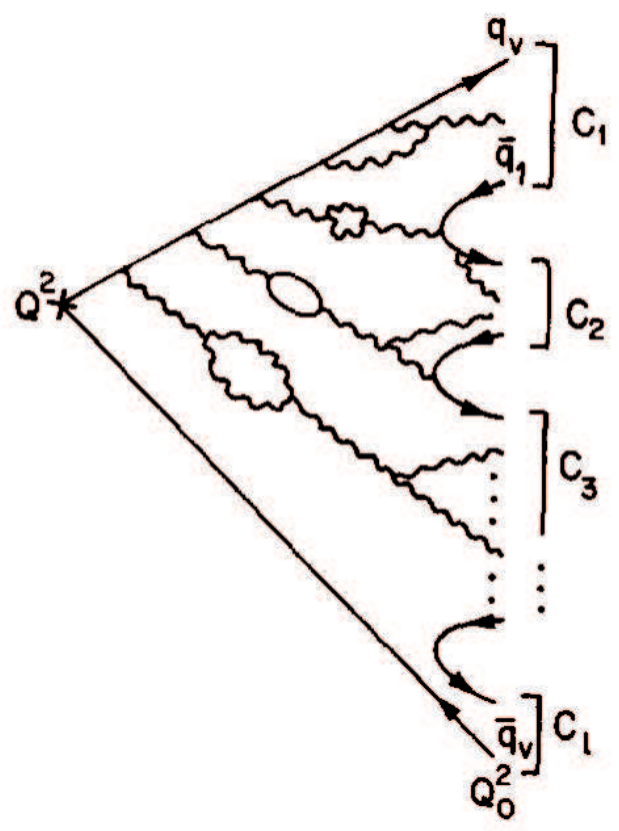

Figure 3: DGLAP shower evolution of the primordial $q \bar{q}$ pair created in $e^{+} e^{-}$annihilation, exhibiting singlet cluster formation at the end [1].

by the requirement that perturbative $\mathrm{QCD}$ is still applicable. After $\mathrm{t}_{0}$ both $\mathrm{Q}^{2}$ gets lower and the time scale of the further evolution slower such that the era of non perturbative QCD begins [5].

However, right at the end of the pQCD phase the shower partons, i.e. quarks and gluons, acquire a peculiar, ordered pattern in space and color-flavour configuration that was called "color pre-confinement" by its inventors, Amati and Veneziano [1]. Fig.3 indicates this ordering of the partons, occuring at $t_{0}$ through peculiarities of the $\mathrm{pQCD}$ matrix elements governing the cascade sub-processes. The cascade thus breaks down into local clusters $c_{i}$ where overall color neutralizes and "hadron-like" flavour combinations predominate; but all of this still in pQCD vacuum. From the virtualities and momenta of the participating partons one can construct the cluster c.m. systems and the (virtual) cluster "masses". This task is performed, e.g. by the pQCD shower Monte Carlo code "HERWIG" constructed by Webber and collaborators [2, 5]. Fig.4 shows the resulting distribution of cluster masses $\mathrm{M}_{c}$, with mean value of about $1.5 \mathrm{GeV}$, and a steep fall-off toward higher virtual masses. By construction under $\mathrm{pQCD}$ governance, these clusters are color singlets, with typical dimension $1 \mathrm{fm}$. Obviously, the stochastic elements of the overall pQCD shower evolution, acting at the level of its various microscopic sub-processes, lead to a rather broad probability distribution of cluster mass and flavour content. Thus arises the first statistical influence on the path toward hadronization.

With this singlet cluster stage we arrive at the end of perturbative QCD applicability, entering non perturbative QCD territory for a picture of the subsequent evolution. Nature, of course, does not hesitate to proceed, which takes place via the parton-hadron phase transition that ends with 


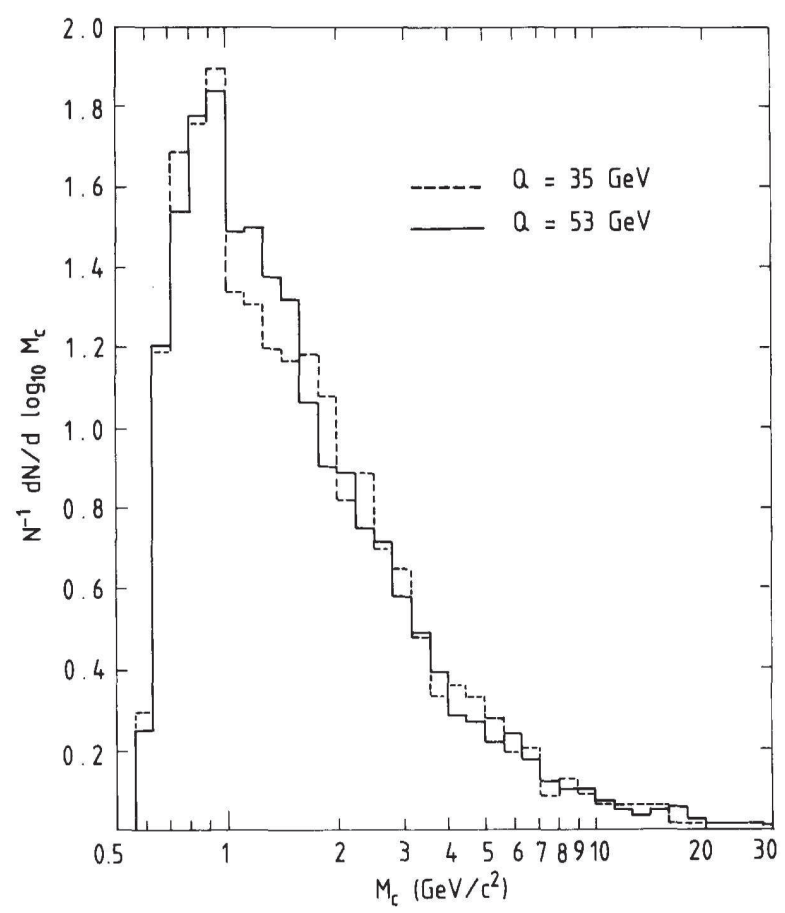

Figure 4: Mass-distribution of color singlet clusters in $e^{+} e^{-}$annihilation to hadrons [2].

confined, on-shell hadrons. It is remarkable, however, to see how smoothly the npQCD models of hadronic structure match with the end product of the pQCD evolution, e.g. dynamical color singlet formation [1]. In $\mathrm{pQCD}$ view these clusters represent local "excitations" of the perturbative vacuum as quantified by a virtual mass; pQCD evolution also suggests that, toward and beyond the cutoff scale $t_{0}$, the time scale of the evolution slows down considerably. It thus appears natural to assume that typical npQCD structures, e.g. quark and gluon condensates, replace the virtual vacuum excitation energy density of the PQCD clusters, given sufficient time (now of order $1 \mathrm{fm} / \mathrm{c}$ ) to develop. We are now well beyond $t_{0}$. In the HERWIG simulation the cluster mass spectrum of Fig.4 is then re-interpreted as a superposition of excited hadronic resonances which subsequently decay quantum mechanically, into the well-known spectrum of low lying on-shell hadrons (and resonances) plus kinetic energy. This decay proceeds under phase space governance, vis a vis the spectrum of hadronic/resonance spins and masses, introducing the finally observed yield distribution among the various hadronic species which reflects their respective spin and phase space weights. Thus ends the QCD evolution in the elementary process of $e^{+} e^{-}$annihilation to di-jets of hadrons. Their pencil-sharp, back to back correlation in space (and in momentum space) clearly represents a highly ordered, low entropy configuration, that results from the strictly back to back configuration of the primordially produced $q \bar{q}$ pair. The subsequent shower period and the eventual decay into hadrons add modest stochastic transverse momentum components, leading to the characteristic "jet cone" topology of the finally observed hadrons. Remarkably, however, the yield distribution among the final hadronic species (which arises from the very same stochastic influences) presents itself as a maximum entropy, equilibrium ensemble, as we infer from the success of the statistical canonical 
ensemble fit to LEP jet hadronization data by Becattini [6], shown in Fig.1.

However: in stressing the dynamical QCD origin of the apparent hadronic species equilibrium (which has been the source of inspiration for Hagedorns development of the statistical hadronization model [18] that has been endlessly challenged until today) I have exaggerated the role of the stochastic elements of the evolution, leading to loss of information. In fact the canonical model fit shown in Fig. 1 wraps up some crucial information, concerning the conditions prevailing at the instant of hadronic freeze-out to on-shell (in vacuum) hadrons and resonances, in its characteristic parameters. Most notably, the derived temperature amounts to $\mathrm{T} \approx 160 \mathrm{MeV}$ here, corresponding to an energy density of about $0.7 \mathrm{GeV} / \mathrm{fm}^{3}$. These values correspond closely, on the one hand, to Hagedorns limiting conditions for a hadronic system [8] and, on the other hand, to modern lattice QCD estimates of the parton-hadron phase transition temperature [23]. These conditions should, in fact, be universal to any hadronizing system, irrespective of its dynamical pre-history. It is thus reassuring to see that the same temperature governs hadronization in central $\mathrm{Pb}+\mathrm{Pb}$ collisions at $\sqrt{s}$ $=17.3 \mathrm{GeV}$, as shown in Fig.2. We conclude that hadronic freeze-out in such reactions occurs in the vicinity of the QCD parton to hadron transition: a consistency check, concerning our above sketch of the QCD evolution toward hadronization. All such high $\sqrt{s}$ hadronization data thus provide for an experimental determination of the parton-hadron phase boundary.

The second parameter of the canonical model employed by Becattini [6] in Fig.1 is the total "fireball" volume contributing the decay hadrons and resonances. In the reaction considered here, $e^{+} e^{-}$annihilation to hadrons at $\sqrt{s}=91.2 \mathrm{GeV}$, it amounts to $27 \mathrm{fm}^{3}$. This value compares well with expectations from the cluster hadronization model by Webber et al. [2, 5] which, at this energy, may feature about 10 clusters on average, of dimension $1 \mathrm{fm}$, decaying simultaneously but independent of each other. Note that in jet hadronization the major fraction of the initial total c.m. energy resides in hadron kinetic energy along the axis defined by the initial $q \bar{q}$ pair.

Finally, the much debated "strangeness under-saturation factor" $\gamma_{s}$ is employed in the canonical fit shown in Fig.1. It represents an additional fugacity factor, inserted for strangeness production into the canonical partition functions, that takes account of the apparent "Wroblewski-suppression" [28] of strangeness relative to light quark production which is characteristic of all elementary collisions. At first sight $\gamma_{s}$ may appear to be merely a fudge factor, within the framework of the canonical model. It indicates that the canonical ensemble of created hadrons deviates from global $\mathrm{u}, \mathrm{d}$, s flavour equilibrium, leading to a relatively modest suppression of $\mathrm{K}$ and $\Lambda$ relative to pions but becoming increasingly effective toward multiply strange hyperon production. Fig. 1 shows that the canonical fit is, in fact, critically constrained by the cascade and omega hyperon data, thus clearly establishing the need of such an auxiliary strangeness fugacity factor, $\gamma_{s}<1$.

Auxiliary, as seen in the hadron/resonance population after hadronization (with which the statistical model has to deal), this apparent strangeness suppression may nevertheless be traced to the pre-history of hadronization. In fact, the QCD evolution, sketched above, consists chiefly of shower formation due to inelastic parton interactions which, studied in isolation, always produce secondaries under governance of strangeness suppression, basically due to the higher strange quark mass which causes a corresponding penalty factor. The emerging pre-hadronic clusters thus contain quarks with a similarly suppressed strangeness fraction. Actually, a similar population pattern arises in the alternative Lund string hadronization picture [4] where the flavour densities u:d:s are predicted to be about $1: 1: 1 / 3$. This relative under-population of strangeness is preserved in the 
eventual cluster decay to on-shell hadrons and hadronic resonances, which redistributes the initial flavour content under governance of the relative phase space weights, as implied by the hadronic mass and spin spectrum. The canonical model fit, which takes a snapshot of the hadron/resonance population right "after birth" $[8,18,19]$, reflects the resulting equilibrium distribution of yields, albeit within the restrictions caused by initial strangeness under-population of the hadron ensemble. Thus, $\gamma_{s}<1$ reflects an absolute strangeness non-equilibrium population which is, however, relatively distributed in statistical equilibrium among the strange hadron species.

In summary we have, at first, stressed the remarkable feature that a hadronic species equilibrium population can arise from fundamental parton interactions which, in other respects, lead to final state configurations (e.g. jets) that are dramatically remote from maximum entropy. The reasons being found in stochastic elements governing the dynamical QCD evolution [1, 2, 3, 4, 5]. However, a more detailed analysis of the key parameters of the canonical ensemble model shows that they preserve critical information about the dynamical pre-history of hadronization: the hadronization temperature and energy density, the system volume prevailing at hadronization, and the strangeness output arising from the preceding microscopic QCD dynamics.

\section{Hadronization and hadronic freeze-out in $\mathbf{A}+\mathbf{A}$ collisions}

In the previous chapter we have concentrated on hadron production in the, perhaps, most elementary process of $e^{+} e^{-}$annihilation to jet hadrons because

1. The observed multiplicity order obeys the canonical equilibrium pattern, initiated by Hagedorn, in the perhaps most striking manner, due to the comprehensive LEP data illustrated in Fig.1.

2. Furthermore, hadronization proceeds into vacuum, on-shell products here, with no essential final state reconfiguration due to inelastic hadronic interaction. Thus hadronization and hadronic freeze-out occur in coincidence.

3. Finally, an elaborate dynamical QCD model exists which features color pre-confinement singlet cluster formation and non-perturbative hadronization, dominated by stochastic elements. This model not only provides plausibility for the canonical ensemble description of the hadronization outcome: it also helps to understand the fundamental parameters of that model, e.g. hadronization temperature, fireball volume, and strangeness under-saturation.

We can now deal with A+A collisions.

\subsection{Hadro-chemical equilibrium}

Turning to hadron formation in nucleus-nucleus collisions, from top SPS to RHIC energy, $17.3 \leq \sqrt{s} \leq 200 \mathrm{GeV}$, Fig.2 illustrates the continuing success of a statistical hadronization model description [11] of NA49 data [29], gathered at $\sqrt{s}=17.3 \mathrm{GeV}$. What is new: the primary hadron multiplicities require $a$ grand canonical ensemble description, 


$$
<n_{i}>=\frac{\left(2 J_{i}+1\right)}{(2 \pi)^{3}} V \int d^{3} p\left\{\exp \left[\left(E_{i}+\mu_{i}\right) / T\right] \pm 1\right\}^{-1}
$$

where $E_{i}^{2}=p_{i}^{2}+m_{i}^{2}$ is the vacuum on-shell energy of species $\mathrm{i}$, and the penalty factor $\exp \left(-E_{i} / T\right)$ is modified by the global chemical potential $\mu_{i}$ for each species, thus taking care of baryon number, strangeness and isospin conservation on average over the entire fireball volume $\mathrm{V}$. We see from Fig. 2 that the hadronic freeze-out temperature $\mathrm{T}=158 \mathrm{MeV}$ is very close to the value derived from canonical ensemble analysis of $e^{+} e^{-} \rightarrow$ hadrons, Fig.1. It also agrees well with the recent predictions of lattice QCD at finite baryo-chemical potential [27], concerning the location of the parton-hadron coexistence line in the $\left[\mathrm{T}, \mu_{B}\right]$ plane. As we shall show below the $\mathrm{T}, \mu_{B}$ values derived from grand canonical analysis [12] of STAR data obtained at top RHIC energy also fall close to this line. One is, thus, tempted to conclude that hadronic species freeze-out does very closely coincide with hadronization, both occuring at the QCD phase boundary, which is thus experimentally located from top SPS energy to top RHIC energy.

Furthermore we recall again that the hadrochemical freeze-out temperature, observed here, agrees with the one observed in the perhaps most simple and "elementary" QCD process of $e^{+} e^{-}$ annihilation to hadrons, where hadronization and hadronic freeze-out coincidence [2, 3]. One is, thus, tempted to suppose that hadronization in central A+A collisions at such high $\sqrt{s}$ should (mutatis mutandis) resemble the QCD evolution sketched in the previous chapter, i.e. shower multiplication of pQCD partons toward decreasing virtuality resulting in a spatial color-flavour correlation ("color pre-confinement") [1] that lends itself to a re-interpretation in terms of non-perturbative QCD singlet clusters whose initial virtual mass and spatial extension converts to "real" hadronic mass and size. The clusters then decay to on-shell hadrons and resonances under governance of phase space and flavour conservation, thus producing the typical "equilibrium" population that is observed, both, in elementary and in A+A collisions. This apparent equilibrium should thus, in both cases, result from the stochastic elements governing the hadron formation process: the hadrons are thus "born into equilibrium" $[8,18,19]$. Right after birth the statistical ensembles provide for a snapshot of these equilibrium distributions. This picture is highly plausible for elementary collisions where the hadrons escape almost instantaneously into vacuum, thus preserving the canonical yield order. Not so in high $\sqrt{s}$ collisions of heavy nuclei. The energy density corresponding to the grand canonical fit of Fig. 2 amounts to about $0.6 \mathrm{GeV}$ per $\mathrm{fm}^{3}$ and, assuming a source radius of $7 \mathrm{fm}$, the source volume will double within about 2-3 fm/c, in a simple isotropic fireball model. At first sight, such an early expansion stage, still at considerable hadronic density, might re-adjust the initial hadronic population ratios via inelastic cascade processes. However such a dynamical cascade evolution of the hadronic equilibrium distribution does, in fact, not occur here. We do observe a freeze-out temperature of about $160 \mathrm{MeV}$, and not the supposed "relaxed" temperature of about $135 \mathrm{MeV}$ that would (with $\varepsilon \propto \mathrm{T}^{4}$ ) govern an equilibrium state at twice the initial volume.

Thus we conclude that somewhat counter-intuitively, an initial hadron/resonance ensemble at $\varepsilon$ $=0.6 \mathrm{GeV} / \mathrm{fm}^{3}$ and $\mathrm{T}=160 \mathrm{MeV}$ stays unattenuated throughout the ensuing hadronic expansion era. In fact it was shown by Bass and Dumitru [30] that (at RHIC energy) a yield distribution derived from hadronization, supposed to occur at an even higher energy density $\left(\varepsilon=1 \mathrm{GeV} / \mathrm{fm}^{3}\right)$ survives the hadronic expansion era (modeled here by the UrQMD transport model) essentially unattenuated. Noting that the QCD parton to hadron phase transformation occurs within the domain $0.6<\varepsilon<1$ 
$\mathrm{GeV} / \mathrm{fm}^{3}$ according to recent lattice QCD, hadronization and dynamical hadronic freeze-out should coincidence closely, in central A+A collisions from top SPS to RHIC energies. We may, thus, indeed learn about matter at the phase boundary.

\subsection{Strangeness enhancement}

The fundamentally new, and unique feature of central A+A collisions is strangeness enhancement. More correctly we are dealing with the fading away of strangeness suppression which is universally characteristic of elementary collisions $[28,31]$. One usually quantifies the strange to light quark density ratio by the "Wroblewski-coefficient" [28]

$$
\lambda_{s}=2(s+\bar{s}) /(u+\bar{u}+d+\bar{d})
$$

which amounts to about 0.25 in $p p, p \bar{p}$ and $e^{+} e^{-}$collisions [31] but to about 0.50 in central $\mathrm{A}+\mathrm{A}$ collisions [11]. This reflects in an increase of about two in the production ratios $K^{+} / \pi$ and $\Lambda / \pi$, from $\mathrm{p}+\mathrm{p}$ to central $\mathrm{Pb}+\mathrm{Pb}$ collisions at top SPS energy [11, 29]. Moreover, suppression or enhancement feature a hierarchy in strangeness number, i.e. the production ratios for $\Xi(s=2)$ and $\Omega(s=3)$ relative to pions increase by about 6 and 15 , respectively [15, 32]. Note that these hyperons contain only a small fraction of the total strangeness yield, due to their small production rate. Their spectacular enhancements thus highlight an overall bulk strangeness increase of about two. Nevertheless they dominate the statistical model fits, due to their long lever arm, as is obvious from Fig. 1 and 2 which also illustrate the overall strangeness enhancement pattern of central A+A collisions: from pions to omegas the yield distribution drops down by a factor of about $0.5 \cdot 10^{-4}$ in $e^{+} e^{-}$annihilation (Fig.1) but only by about $0.8 \cdot 10^{-3}$ (average of omega and anti-omega) in central $\mathrm{Pb}+\mathrm{Pb}$ collisions (Fig.2).

At the level of the statistical model description the hadron multiplicity data for central heavy nucleus collisions are universally well described by the grand canonical hadron/resonance ensemble, from top AGS to top RHIC energies [11, 12, 13, 14, 15, 18, 19]. Fig.2 represents a typical example [11] obtained at top SPS energy; the NA49 data employed here [29] represent the total yields in $4 \pi$ acceptance which, even in a large acceptance detector system, require significant extrapolation into unmeasured regions of phase space which are, moreover, different for the various species, thus giving rise to a species-dependent systematical uncertainty ranging from 10 to $30 \%$. In view of such experimental conditions the fit quality (with $\chi^{2}=1.8 / d o f$ ) is quite satisfactory. This also supports the significance of the notorious strangeness damping factor $\gamma_{s}=0.83$, employed here: it suppresses the omega yields by a factor of 0.56 , well in excess of systematical errors. We will return to second order corrections, such as this, in chapter 4 . The Wroblewski factor, corresponding to this fit, is $\lambda_{s}=0.52$.

A grand canonical fit to recent RHIC data (central collisions of $\mathrm{Au}+\mathrm{Au}$ at $\sqrt{s}=200 \mathrm{GeV}$ ) by Andronic, Braun-Munzinger and Stachel [12] is shown in Fig.5. These authors prefer to base the grand canonical fit on hadron multiplicity ratios which are determined at mid-rapidity owing to the limited acceptance of the STAR and PHENIX experiments. We see, first of all, an omega to pion ratio of $10^{-3}$ in close agreement with the NA49 data of Fig.2. All anti-hadron to hadron ratios (for a given species) fall close to unity, attesting to a small mid rapidity excess of quarks over anti-quarks 


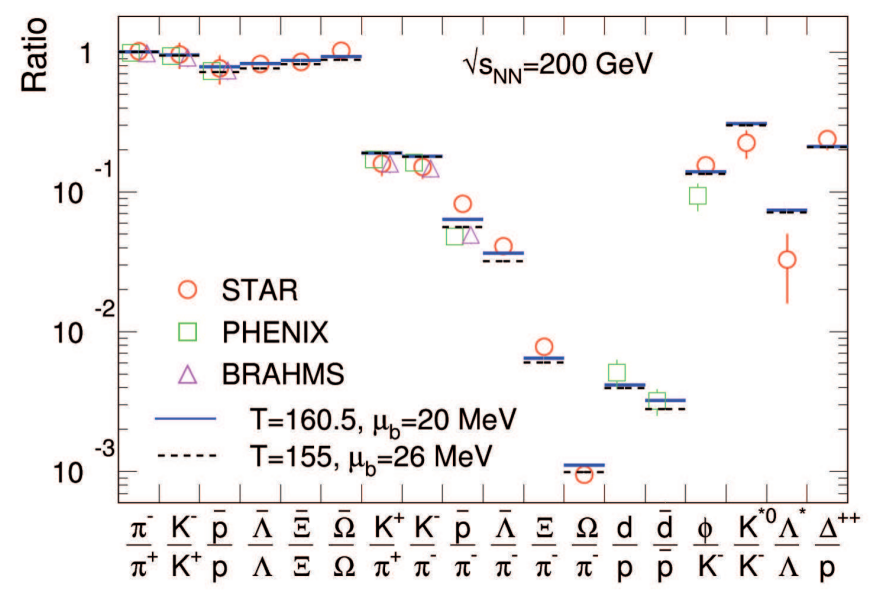

Figure 5: Hadron production ratios at midrapidity in central $\mathrm{Au}+\mathrm{Au}$ collisions at RHIC, $\sqrt{s}=200 \mathrm{GeV}$, and predictions by the grand canonical model [12].

(as reflected in a small baryochemical potential, $\mu_{B} \approx 20 \mathrm{MeV}$ ) - in contrast to the situation encountered at $\sqrt{s}=17.3 \mathrm{GeV}$ where $\mu_{B} \approx 250 \mathrm{MeV}$ (Fig.2). Most remarkably, the derived hadronization temperature, of $\mathrm{T}=160 \pm 5 \mathrm{MeV}$, agrees perfectly well with the value observed at $\sqrt{s}=17.3 \mathrm{GeV}$ and, moreover with the one observed in $e^{+} e^{-}$annihilation at $\sqrt{s}=91.2 \mathrm{GeV}$ (Fig.1). This temperature thus appears to be universal to hadronization at high $\sqrt{s}$. Furthermore it coincides with the "critical" temperature as derived by lattice QCD [22, 27], to prevail at the parton-hadron phase boundary. Thus there is no evidence for a phase of statistical dynamical inelastic equilibrium, occuring subsequent to hadronization in $\mathrm{A}+\mathrm{A}$ collisions [21, 22] - in $e^{+} e^{-}$annihilation there is no such phase by definition. Hadronization and hadro-chemical freeze-out thus occur in very close proximity at such high $\sqrt{s}$. We note, however, that this simplicity is lost toward the lower SPS, and notably the AGS energy regimes, where one encounters grand canonical hadronic freeze-out temperatures as low as $125 \mathrm{MeV}$ [11, 12], falling far below the QCD phase boundary expected from recent lattice studies at a high baryo-chemical potential [27]. This indicates a qualitatively different expansion dynamics [21, 22], to which we return in sect. 4.3.

\subsection{Origin of strangeness enhancement}

According to our above argument strangeness enhancement in central A+A collisions should be due to a mechanism located in the dynamics prior to, or during hadronization, which removes (in part) the strangeness suppression characteristic of elementary collisions. This latter has oftentimes been associated with the conditions prevailing in a small volume, i.e. both with the constraints imposed by a strict, local conservation of quantum numbers, and with the higher penalty factor resulting from the higher mass of the strange - anti-strange flavour pair - both mechanisms together restricting the accessible phase space for creation of secondary particles. In the framework of hadronization, outlined in sec. 2 for $e^{+} e^{-}$annihilation to hadrons, this "samll volume" situation is captured in the form of narrow local spatial clusters of quark-gluon color-preconfinement which set the stage for hadronization. In the Webber [2] version of this model one expects to find about 10-15 
such clusters at $50 \leq \sqrt{s} \leq 90 \mathrm{GeV}$, which are isolated in vacuum and hadronize independent of each other. We note, besides, that Becattini and Passaleva [7] have shown that, for a Lorentz invariant observable such as hadron multiplicity, these hadronization subvolumes (which are boosted with respect to each other) can be treated additively as a single, combined source. This computational trick does, however, not affect the relative hadronic composition which is still characteristic of the single cluster quantum number constraints, prevailing in the non-overlapping individual clusters.

However, now turning to $\mathrm{A}+\mathrm{A}$ collisions at high $\sqrt{s}$, it is obvious that their typical conditions, of a high energy and parton collision frequency density [26] occupying a large primordial interaction volume, might lead to considerable spatial cluster overlap. In fact the naive expectation that, at RHIC energy, $\sqrt{s}=200 \mathrm{GeV}$, each interacting initial nucleon pair might likewise create about 15 pre-hadronization clusters we would expect a total of about 3000 such clusters in a central $\mathrm{Au}+\mathrm{Au}$ collision. The implicit assumption that the initially created partonic pQCD showers still develop independent of each other, in this situation, is probably inadequate, such that inter-shower multiplication of secondaries will lead to a further increase of cluster spatial density. Assuming a single cluster volume of about $1.5 \mathrm{fm}^{3}$ [3] we would infer, from such naive arguments, that non-overlapping clusters would occupy a total volume in excess of $5000 \mathrm{fm}^{3}$, much in excess of the expected total interaction volume prevailing during the time interval of 2-3 fm/c, after the end of pQCD shower evolution [3]. We thus expect considerable cluster overlap, to extended super-clusters: a large, quantum number coherent volume is born, far in excess of confinement dimension. It will develop toward hadrons under global, non-local quantum number conservation, much like any other quantum mechanically coherent object, and its decay will proceed via a QM decoherence transition. I.e. its decay products will be in a quasi-classical state, the "quasi" reminding us that this state contains hadronic resonances as well as on-shell hadrons $[8,17]$. This hadron-resonance "gas" will occupy phase space uniformly (for a given total volume and temperature) owing to the stochastic factors influencing both the preceding QCD evolution, and the eventual decay. It is, thus, not surprising that this state is well represented by a quasi-classical grand canonical Gibbs ensemble [18, 19, 20]. In fact, the third fundamental parameter of this ensemble (besides $\mathrm{T}$ and $\mathrm{V}$ ) is the global chemical potential $\mu$ that represents the fact that quantum number in the preceding decoherence decay was preserved globally, i.e. only on average over the entire volume. We wish to re-iterate that the GC ensemble, by itself, merely captures a snapshot of the system right after its formation. It has been the aim of the above line of argument to show that a plausible QCD evolution can be conceived that leads, exactly, to the conditions pictured in the statistical GC model. In fact, this model provides for a satisfactory representation of the strangeness enhancement phenomena.

The above picture, of pre-hadronization singlet cluster overlap in A+A collisions reminds one of a percolation situation [26]. In fact, the degree of cluster overlap should increase with $\sqrt{s}$, mass number A (i.e. with an exponent between $1 / 3$ and 2/3) and collisions centrality. The resulting expectation that, in general, the Wroblewski ratio $\lambda_{s}$, and in more detail certain characteristic hadronic yield ratios reflecting the relative strangeness to non-strangeness output, should exhibit a smooth rise (toward saturation at the GC level) within the above variables, has been verified experimentally $[25,26,32,33,34,35]$. Most experiments have provided data for the dependence on centrality in $\mathrm{A} \approx 200$ collisions $[32,33,34,35]$, of hadronic production ratios such as $K / \pi, \Lambda / \pi, \Xi / \pi$ and $\Omega / \pi$, confronting them with a base-line of various elementary collision yield ratios, extracted from $p+p, e^{+} e^{-}$and $p+B e$ data at similar energy. In addition NA49 has measured the $K / \pi, \Lambda / \pi$ and 


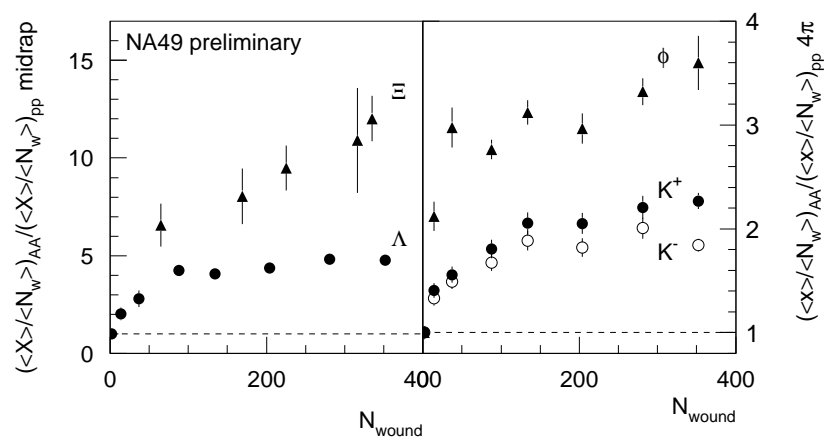

Figure 6: Enhancement of the $\Lambda, \Xi, \Phi$ and $K$ yield per wounded nucleon [25,34] as a function of collision centrality in $\mathrm{Pb}+\mathrm{Pb}$ at $\sqrt{s}=17.3 \mathrm{GeV}$.

$\Phi / \pi$ ratios in central collisions of light nuclei, ${ }^{12} \mathrm{C}+{ }^{12} \mathrm{C}$ and ${ }^{28} \mathrm{Si}+{ }^{28} \mathrm{Si}$, at various SPS energies $[25,32,34]$. These data are usually displayed on a common scale of "participating' or "wounded" nucleon number as derived, for each collision geometry, by Glauber simulation.

We illustrate typical examples of such studies in Fig.6 and 7. Fig.6 shows SPS results of NA49 $[24,33]$, the left hand panel giving the $\Lambda$ and $\Xi$ production rate per wounded nucleon in various $\mathrm{A}+\mathrm{A}$ collision geometries, as normalized to the corresponding $p+p$ yields $\left(N_{W}=2\right.$ by definition). The data shown here refer to the respective hyperon yields at mid-rapidity. The right hand panel shows the $K / W$ and $\Phi / W$ ratios vs. participant number, for the same collisions and geometries, but it employs the extrapolation to total $4 \pi$ of the corresponding yields. Fig.7 (left panel) shows similar data at top RHIC energy obtained by the STAR experiment [34] for the $\Lambda$ and $\Xi$ hyperons and their antiparticle partners, in a plot similar to that of Fig.6 (left panel), exhibiting the yields per participant in $\mathrm{Au}+\mathrm{Au}$ collisions at various centralities, as normalized to the corresponding $p+p$ yields (all taken at mid-rapidity).

We conclude that (irrespective of the different representations of the above data) all strange or multi-strange hadronic species exhibit a smooth rise (different in detail) of production yield (relative to the strangeness suppression situation characterizing small volume elementary collisions) with increasing interaction volume as offered by the primordial reaction geometry. However, it is important to note that it is not the "large volume" per se that causes the transition from small volume, micro-canonical or canonical strangeness suppression to grand canonical strangeness saturation. Two large interacting clouds of a dilute nucleon gas would, of course, yield exactly the $p+p$ particle ratios. It is the coincidence of extended volume and high energy density, typical of A + A collisions at high $\sqrt{s}$, that causes the effect. Such conditions will, in fact, lead to an increasing overlap of QCD pre-hadronization singlet clusters, as discussed above, thus creating large, coherent sub-volume sections. Actually, this description of the dynamical evolution may also imply an appropriate model for "Quark Gluon Plasma" creation.

We see in Fig. 6 that the $K$ and $\Lambda$ yields (which represent the Wroblewski coefficient $\lambda_{s}$ ) ascend steeply, at first, with system size but turn into saturation already for collision systems of about 60-100 participants, corresponding to semi-peripheral collision geometry in $A=200$ nuclear interactions. For cascade hyperons saturation occurs at higher collision volume. This behaviour 

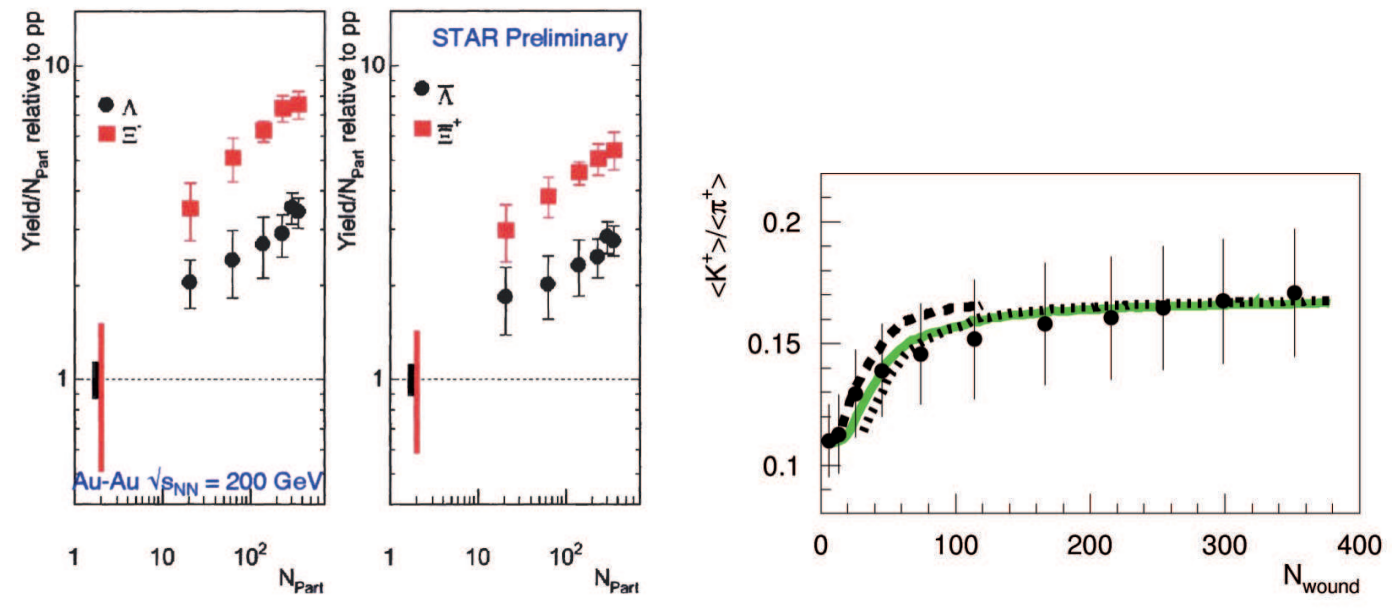

Figure 7: $\Lambda$ and cascade hyperons and their antiparticles: increase of the midrapidity yield per participant with centrality in $\mathrm{Au}+\mathrm{Au}$ at $\sqrt{s}=200 \mathrm{GeV}[32,35]$.

would indeed follow from an increasing degree of cluster overlap occuring with collision centrality, with percolation super-cluster of increasing size being formed. The statistical model also predicts a steep increase toward unity of the strangeness suppression factor $\eta$, with increasing coherent volume [15, 36]; the GC situation can thus be established as the large volume limit of the canonical ensemble. In ref. [15] is it also shown that $\eta$ approaches unity (strangeness saturation) more slowly with increasing hadronic strangeness. Thus, at first sight there appears to be perfect overall harmony between data and statistical model. However, a final step is missing. For $s=1$ one obtains $\eta \rightarrow 1$ already at a remarkably small volume [15], of about $60 \mathrm{fm}^{3}$, and even $\mathrm{s}=2$ saturates at about $250 \mathrm{fm}^{3}$. Whereas the participant or wounded nucleon numbers where these saturations appear to occur in top SPS to RHIC data are as high as about 80 and 250, respectively, and the saturation transition occurs more slowly with centrality. Now, $60 \mathrm{fm}^{3}$ is not the "fireball" eigenvolume of about 80 interacting nucleons: the relationship between experimental $N_{\text {part }}$ and statistical model V is not straight forward.

However, such a relationship can be established by means of a simple percolation model [26] which quantifies the naive expectation that super-cluster coalescence will develop gradually, with increasing average energy density that should, by itself, also exhibit a smooth relationship to collision centrality as monitored by $\mathrm{N}_{\text {part }}$. I.e. at first there will be several, relatively small independent super-clusters hadronizing separately, which amalgamate gradually with increasing centrality. Thus the participating nucleon induced shower cascades, and their resulting local singlet clusters will not, right onward from peripheral collisions, feed into a single super-cluster with volume proportional to participant nucleon number. Thus super-cluster size is not directly proportional to $\mathrm{N}_{\text {part }}$ : it somehow lags behind. The percolation model (for details see ref. [26]) thus determines the super-cluster size and number distribution, in bins of increasing centrality, then to determine the appropriate strangeness suppression factors $\eta_{i}$ for each super-cluster $i$ according to the statistical model $[15,36]$. The final strangeness output results from a weighted average of all individual super-cluster contributions. A typical result is shown in Fig.7 (right hand panel), which illustrates the $K^{+} / \pi^{+}$production ratio vs. $N_{W}$ as obtained by PHENIX [37] in Au+Au collisions at $\sqrt{s}=200$ 
$\mathrm{GeV}$. The result of the combined statistical and cluster percolation model gives a perfect account of the relatively slow rise toward strangeness saturation. Also included is a prediction for the $\mathrm{Cu}+\mathrm{Cu}$ collisions studied more recently at RHIC, which rises more steeply with $\mathrm{N}_{W}$. This illustrates the indirect relationship between $\mathrm{N}_{\text {part }}$ or $\mathrm{N}_{W}$ and the corresponding size of super-clusters: at $N \approx 100$ the $\mathrm{Au}+\mathrm{Au}$ collision system exhibits a typical peripheral geometry, with extended contributions from dilute surface density regions (in which participating nucleons create relatively small energy densities) whereas the $\mathrm{Cu}+\mathrm{Cu}$ collision is already near central, thus featuring a higher average energy density.

We conclude that the overall body of strangeness enhancement phenomena, typical of A+A collisions, can be understood in a picture of amalgamating partonic singlet clusters that form at the end of a preceding pQCD DGLAP shower evolution, at high $\sqrt{s}$, which we have adapted here from previous QCD studies of jet hadronization in the elementary $e^{+} e^{-}$annihilation reaction $[1,2,3,4,5]$. It appears that the above line of argument, as suggested in the introduction, can indeed establish a direct link between the relatively straight forward QCD analysis of jet hadronization, and the more complicated dynamics of A+A collisions which gives rise, among other features specific to such collisions, to the phenomena of strangeness saturation. Within this line of argument the success of a statistical model description of the relative hadronic yield patterns, i.e. the mysterious apparent equilibrium distribution, can be also understood in detail, as a result of the preceding dynamical evolution.

\section{Problems}

In the preceding chapters we have oftentimes ignored to face, in detail, certain problems and objections, such as the extra fugacity factor $\gamma_{s}$ or the problem of employing $4 \pi$ or midrapidity data, etc.. We have thus given only an overall, qualitative description of hadronization - but exactly this was our purpose. We shall now first turn to the midrapidity problem which, as it turns out, is partially related to the $\gamma_{s}$ question, and end with a brief discussion of hadronization specifically at lower SPS and AGS energies where our picture of an initial pQCD parton shower evolution can not be expected to be valid.

\subsection{Midrapidity vs. $4 \pi$ data}

We start our consideration from two simple, limiting situations: at very low energy, i.e. in the $\sqrt{s}=1-2 \mathrm{GeV}$ domain of the Bevalac and SIS experiments, the entire rapidity distribution resides in a gap $\Delta_{y}<2$. A single spherical fireball at a temperature of $80 \mathrm{MeV}$ spreads its particles over approximately $\delta_{y}=1.4$ units, i.e. it essentially fills the entire rapidity gap, the experimentalists having a hard time removing, at least, the spectator particles, but they will effortlessly cover the rather narrow $\delta_{y}$ interval. Thus, essentially all existing data are extrapolations to $4 \pi$, and smaller intervals are difficult even technically because of the "banana-shaped" typical fixed target spectrometer acceptances in the plane $\left(y, p_{T}\right)$. On the contrary, at extremely high energy (i.e. perhaps at the LHC energy $\sqrt{s}=5.5 \mathrm{TeV}$ ) the $y$-distribution becomes boost invariant over about 10 units of rapidity, such that the surface "corona" contribution (from single collisions in the Woods-Saxon surface) which will still reside in the vicinity of $\left|Y_{\text {beam }}\right|$ becomes a very small fraction of the total 
cross section. With essentially flat rapidity distributions the acceptance does not matter much, and one will concentrate on midrapidity data where all detector acceptance overlap.

Unfortunately, however, all data discussed at present, from AGS to RHIC energy, fall unhappily in between these extremes. From AGS to top SPS energies the rapidity gap widens to $\Delta y=3-6$, too wide to be described as the decay outcome of a single central fireball as the interaction volume gets stretched by preponderance of longitudinal over transverse expansion flow. Moreover both here, and up to top RHIC energy, the rapidity distributions differ for the various hadronic species, and are far from boost invariance flatness [38]. Thus, midrapidity hadron yield ratios are systematically different from $4 \pi$ ratios. An example of this difficulty is given by the SPS data shown in Fig. 6 . The left hand panel illustrates the midrapidity ratio (per wounded nucleon) of the $\Lambda$ yield in $\mathrm{Pb}+\mathrm{Pb}$ collisions, relative to the one observed (again at midrapidity) in $\mathrm{p}+\mathrm{p}$ collisions at the same energy. Noting that the $\Lambda$ distribution features even a minimum at midrapidity for the latter system, it is no surprise to see the yield ratio ascending to a value of about 4.5 with increasing centrality. Whereas the $K^{+}$data in $4 \pi$ geometry (right panel) exhibit an increase with $\mathrm{Pb}+\mathrm{Pb}$ collision centrality, of only a factor of about two, over the $\mathrm{p}+\mathrm{p}$ reference point that also refers to $4 \pi$ extrapolated data here. As the $\Lambda$ and $K^{+}$yields both represent the major fractions of the s and anti-s production rates, respectively, and should thus reveal a similar bulk strangeness grand canonical saturation pattern, we are faced with a serious apparent inconsistency. We have disregarded this in ch. 3 because there our emphasis was to demonstrate, first of all, the basic phenomena of relative strangeness increase with system size.

Before rushing to conclusions let us analyze the origin of the above difficulties which have resulted in a vivid but premature controversy among the various schools of statistical model proponents $[11,12]$. To this end we note, first, that the origin of the above, and other apparent incongruencies resides in the fact that $t w o$ closely interwined mechanisms govern hadron formation, in proceeding from elementary $\mathrm{p}+\mathrm{p}$ (or $\mathrm{p}+\mathrm{Be}[32]$ ) to central $\mathrm{A}+\mathrm{A}$ collisions. These mechanisms are well separated on the overall time scale. Primordial baryons, and their net baryon number content, are disentangled in the course of initial pQCD interaction. In dependence on $\mathrm{A}, \sqrt{s}$ and reaction geometry a specific re-distribution arises in longitudinal phase space, both of resulting net baryon number and energy density. This distribution depends on the characteristic "stopping power", as offered by the longitudinal thickness of the reactants. The higher the stopping power, the wider the shift of the incident valence quarks, away from initial $y_{\text {proj }}$ and $y_{\text {targ }}$ and toward midrapidity alongside with the buildup of high energy density.

At later times the clusters or super-clusters that approach hadronization are differently composed according to their location in $y$ space. Thus, for example at top SPS energy, there arises a hierarchy of net baryon $y$ distribution shapes: from maximally forward/backward peaked in minimum bias $\mathrm{p}+\mathrm{p}$ collisions to near "flat top" shape in central $\mathrm{Pb}+\mathrm{Pb}$. This reflects in the y-distribution of the (net) proton, $\Lambda, \Xi$ and $K^{+}$yields which tend to be much broader than those of the corresponding anti-hadrons which are free of initial $u, d$ quarks. The result: evaluated in small bins of rapidity all hadron ratios depend on the local rapidity. However we need to recall here that the smallest bin size suitable for statistical model analysis is given by the spread in rapidity resulting from the decay of an ideal single, isolated fireball which, at a hadronization temperature of $160 \mathrm{MeV}$, amounts to $\Delta_{y} \approx 2$ [11]. The relatively narrow rapidity intervals, populated at AGS and lower SPS energies, thus do not allow for an analysis in several separate bins, more evidently so as we have to recall that 


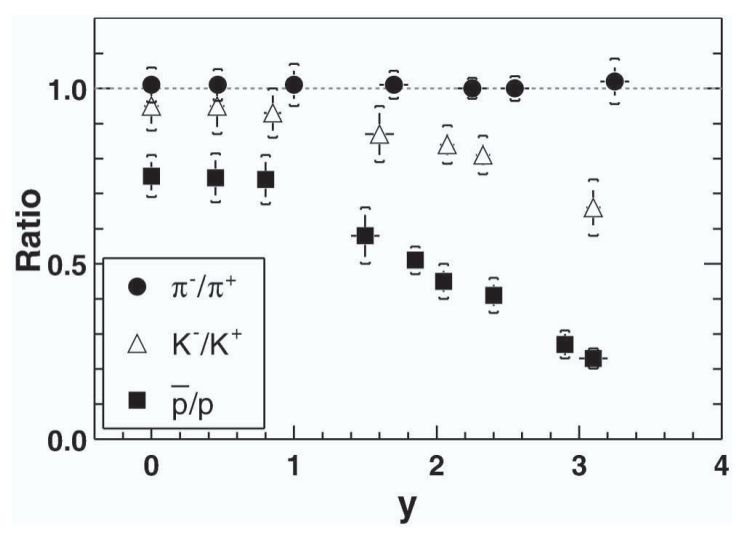

Figure 8: Hadron production ratios vs. rapidity, central $\mathrm{Au}+\mathrm{Au}$ collisions at $\sqrt{s}=200 \mathrm{GeV}$, BRAHMS experiment [38].

the physics of equal mass target and projectile collisions exhibits reflection symmetry about midrapidity. One is thus left with only two options, to analyze either in the interval $\left(y_{\text {mid }} \pm 1.0\right)$, or in $4 \pi$ , both options not ideal - thus the vivid controversy concerning a correct statistical model approach $[11,12]$. We shall illustrate this situation in the next section, devoted to the $\gamma_{s}$ problem, but conclude, in the meanwhile, that statistical model analysis retains at present a certain approximative character at intermediate $\sqrt{s}[11,13]$.

However, at top RHIC energy the wider rapidity gap, $\Delta_{y} \approx 10$, (or even its half, because of reflection symmetry) permits a statistical model analysis which is differential in $y$. This fascinating new aspect has been discussed by Röhrich [39], based on wide acceptance Au+Au collision data gathered by the BRAHMS detector [38], which are shown in Fig.8. Illustrating the remarks above concerning the stopping power effect on the net baryon number rapidity distribution, the figure shows a dramatic dependence of the antiproton to proton ratio: a short "plateau" region (a la boost invariance) governs the interval $y_{C M} \pm 1$; this is also true for all other hadron yields and ratios, measured here and by the PHOBOS and STAR experiments at RHIC. But at $y_{C M}>1$ the $\bar{p} / p$ ratio drops down steeply toward 0.2 at $y \approx 3.5$, thus making close contact to the top SPS energy values obtained at midrapidity by NA49 [40]. The $K^{-} / K^{+}$ratio follows a similar drop-off pattern, to about 0.65 , again matching closely with the top SPS energy value, of about 0.6 [29]. The deviation from unity of these ratios reflects the density on initial valence $u, d$ quarks, relative to the density of newly created light and strange quark - anti-quark pairs, i.e. the net baryon number density and its rapidity distribution. Exactly this density ratio defines the baryo-chemical potential parameter of the GC ensemble. Thus, in analyzing successive bins of these rapidity distributions, the major variation in the GC fit concerns the baryo-chemical potential $\mu_{B}$ which increases from about $20 \mathrm{MeV}$ (see Fig.5) at midrapidity, to about $150 \mathrm{MeV}$ at $y \geq 3$ [39], while the hadronization temperature stays near constant, at $\mathrm{T}=160 \pm 5 \mathrm{MeV}$.

Referring to [38, 39] for detail we conclude, first, that statistical model analysis can be carried out differentially in $y$ at top RHIC energy. Remarkably, the total rapidity interval is just wide enough to permit such an analysis, but it is narrow enough, on the other hand, to establish a situation which is quite different from ideal boost invariance; such that local hadron composition varies with 
the site of hadronization, in longitudinal phase space.

We are thus on firmer grounds, concerning statistical model analysis at RHIC energy and beyond. Moreover, the above RHIC observations are highly relevant for the general topic of hadronization in $\mathrm{A}+\mathrm{A}$ collisions at high $\sqrt{s}$. We see that hadronization occurs "late", and not from a single, globally coherent fireball volume but from a sequence of super-clusters with limited internal rapidity spread, and with a valence quark density which changes along the y axis according to the primordial stopping process. We have shown in sect.3.4 that grand canonical strangeness saturation does not require all 400 participant nucleons to gather in a single super-cluster (Fig.6), and ref. [15] suggests that $N_{\text {part }}=40-80$ suffices, even for s=3. It will be interesting to see how these phenomena evolve at LHC energy.

One disturbing feature remains in the overall picture. The STAR data [35] illustrated in Fig.7 (left frame) do not really exhibit the saturation pattern predicted by the model [15]. The hyperons yields per participant keep increasing all the way up to fully central collisions, and this is also true for the SPS NA49 cascade hyperon yield [34] shown in Fig.6 (left frame), whereas the s=1 yields do indeed exhibit a saturation pattern. Could this imply that full strangeness saturation of $\mathrm{s}=2$ and 3 hyperons does not really occur? In the light of the above discussion of a hadronization situation with successive super-clusters, ordered in rapidity and decaying each by themselves, we might be tempted to conclude that they stay too small, in such a situation of extreme longitudinal expansion flow. The ongoing analysis of RHIC data at $\sqrt{s}=64 \mathrm{GeV}$ might clarify this. Or should we propose [41] that the extra suppression factor $\gamma_{s}$ equals unity only in fully central collisions at RHIC, dropping down with smaller participant number? We are apparently exchanging one evil by the other - to which we shall turn next.

\subsection{The strangeness suppression factor Gamma}

The question whether an extra fugacity factor $\gamma_{s}$ suppressing strange hadronic species (which is familiar from canonical model studies of elementary collisions) is required in the GC approach is still open. Furthermore even if this question is to be answered affirmatively the origin of a $\gamma_{s}<1$ is not uniquely understood $[11,12,14]$. Let us first try some simple considerations. The problem could be an off-shot of the above $4 \pi$ vs. midrapidity discussion. We note that an ideal single fireball at $\mathrm{T}=160 \mathrm{MeV}$ creates a much broader rapidity distribution for $\pi$ than for $\Omega$, the reason being the decrease with hadron mass of the average thermal velocity, of which $y$ represents the longitudinal component. Thus $\sigma(y)$ of a Gaussian parametrization decreases by more than a factor of two. Thus, in a relatively narrow window centered at midrapidity one records a higher fraction of the total $\Omega$ yield, as compared to the pion yield fraction. I.e. the $\Omega / \pi$ ratio is higher at midrapidity than in $4 \pi$ perhaps by a factor of two [11] for a midrapidity window of $\Delta y=1$. To a somewhat lesser extent this repeats for the cascade, $\Lambda$ and $\Phi$ to pion ratios. We thus get a spurious extra strangeness enhancement, increasing toward $s=2$ and 3 , with midrapidity data, at the SPS (narrow gap). However this argument is an oversimplification as hadrons are not produced from an ideal single isotropic fireball even at SPS energy.

Nevertheless, this effect is indeed observed. Fig.9 shows a GC fit by Becattini [42] employing a $\Delta y=1$ midrapidity cut on the NA49 data that result [11] in the fit of Fig.2, in their $4 \pi$ version. The latter fit requires $\gamma_{s}=0.83$ with adequate statistical significance whereas the midrapidity fit procedure gives $\gamma_{s}=0.96 \pm 0.04$, compatible with unity, and in agreement with the parallel midrapidity 


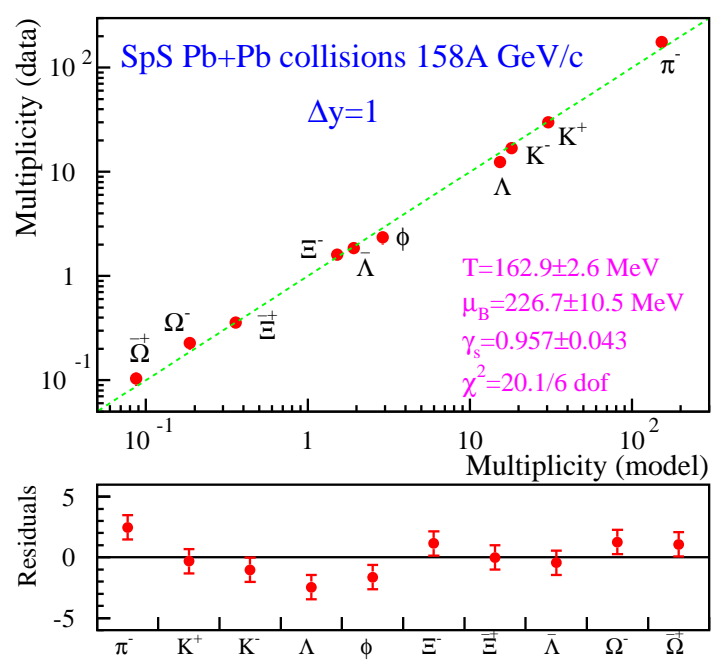

Figure 9: Midrapidity hadron multiplicities in central $\mathrm{Pb}+\mathrm{Pb}$ collisions at $\sqrt{s}=17.2 \mathrm{GeV}$ [25,29] vs. grand canonical model prediction by Becattini $[11,42]$.

analysis of Braun-Munzinger, Stachel and collaborators [12] which employs $\gamma_{s}=1$, throughout. We have shown their RHIC data analysis in Fig.5. Comparing Figs. 2 and 9 we see that the freezeout temperature, $\mathrm{T}=(160 \pm 3) \mathrm{MeV}$, is insensitive to the alternative choices, whereas the derived value of the baryo-chemical potential $\mu_{B}$ is significantly lower at midrapidity than in $4 \pi$. This reminds us of the stopping power effects discussed in sec.4.1. Already at top SPS energy the final rapidity distribution of the initial $\mathrm{u}, \mathrm{d}$ valence quarks from the target and projectile nuclei (which defines the net baryon number distribution) exhibits a slight minimum at midrapidity, as is reflected directly in the corresponding net proton and net $\Lambda$ distributions. As a result the y-distributions of $\mathrm{p}$ and $\Lambda$ are drastically different from the corresponding anti-hadron distributions, which are Gaussian about midrapidity. To a lesser degree this also causes the y distributions of all other valence quark carrying hadrons (notably $K^{+}$and $\Xi$ ) to be broader than those of their respective anti-hadron partners. Again, it is clear that a relatively narrow midrapidity cut will lead to hadron production ratios which are different from the corresponding $4 \pi$ values. To assess the possible consequences of this phenomenon Becattini [42] has formerly performed a GC analysis of a subset of then preliminary $\mathrm{NA} 49 \mathrm{~Pb}+\mathrm{Pb}$ central collision data, only retaining hadrons that consist entirely of newly created quarks. The corresponding fit is shown in Fig.10 and, within the limitations of a poor statistical significance, we take note that $\gamma_{s}$ again results at unity whereas the temperature stays unchanged throughout the sequence of Figs.2,9 and 10. We note that the preliminary NA49 data, employed here, have been since corrected (lowering the $\bar{p}$ and $\Omega$ yields to the final values employed in Figs. 2 and 9 which would improve the significance). This exercise has thus to be repeated, and extended toward the lower RHIC energies. We have illustrated it here to register the interesting idea.

In conclusion it appears that the principal difficulties, discussed in this and in the preceding 


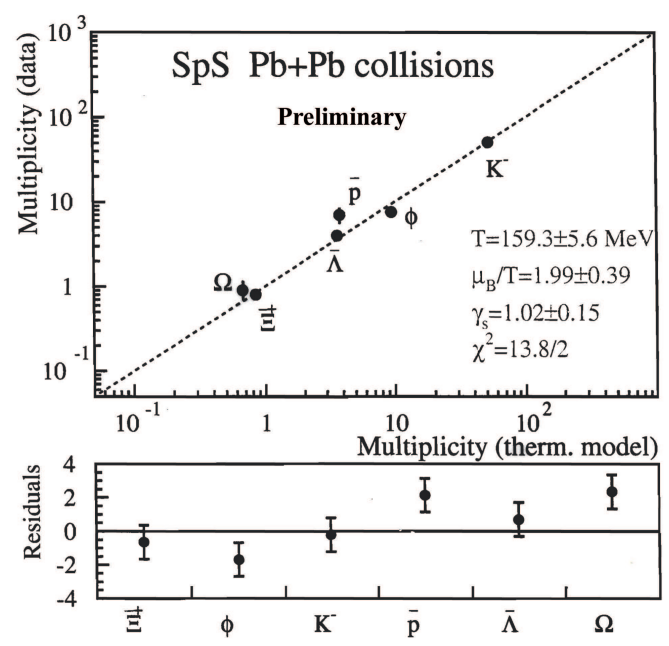

Figure 10: Preliminary grand canonical model fit to central $\mathrm{Pb}+\mathrm{Pb}$ collision data in $4 \pi$, at $\sqrt{s}=17.3 \mathrm{GeV}$, where only valence-quark-free hadrons are included [42].

section, might prohibit an ideal, carefree definition for the proper employment of GC analysis at intermediate $\sqrt{s}$. The $\gamma_{s}$ problem can, thus, not find a satisfactory answer as of yet. This situation will change with the advent of LHC data where we can expect to observe more nearly boost invariant rapidity distributions.

\subsection{Grand Canonical analysis at lower $\sqrt{s}$}

We have seen in sect.4.1 and 4.2 that the simple GC multiplicity analysis with global parameters resides on an idealization of the collision which cannot exactly fit physical reality, and discrepancies are thus to be expected. Thus a new mechanism or a modification of the basic scheme proves to be relevant only if it leads to a major improvement of agreement with the data. This refers, among others, to the question whether a determination of the (partonic phase) strange to non-strange density ratio before hadronization is possible, and reflected in the $\gamma_{s}$ factor. This would take into account that at high $\sqrt{s}$ this ratio might be smaller in a quark gluon phase than in an equilibrium GC hadronic ensemble. This expectation remains unverified as of yet, and likewise the proposal [43] that hadronic freeze-out occurs, not to a quasi-classical but to a mean field situation which implies in-medium modified hadron masses. Besides being somewhat counterintuitive (after freeze-out the hadrons should be on-shell) this proposal introduces numerous further parameters but with a modest net gain in fit significance.

However, the fit variations in Figs.2,5,9,10 do indeed illustrate the general observation that the implied freeze-out temperature stays rather insensitive to such second-order modifications, contrary to the claims made in ref. [12]. In fact, from top SPS energy $\sqrt{s}=17.3 \mathrm{GeV}$ via RHIC at 64,130 and $200 \mathrm{GeV}$ the temperature is compatible with a constant, global average value of $165 \pm 5 \mathrm{MeV}$ $[11,12,38]$. This value does remarkably well coincidence with recent lattice QCD predictions of the critical QCD temperature, both at $\mu_{B}=0$ [23] and at finite $\mu_{B}$ [27]. Toward lower $\sqrt{s}$, the derived freeze-out temperatures drop down smoothly until SIS energy, permitting an interpolating 


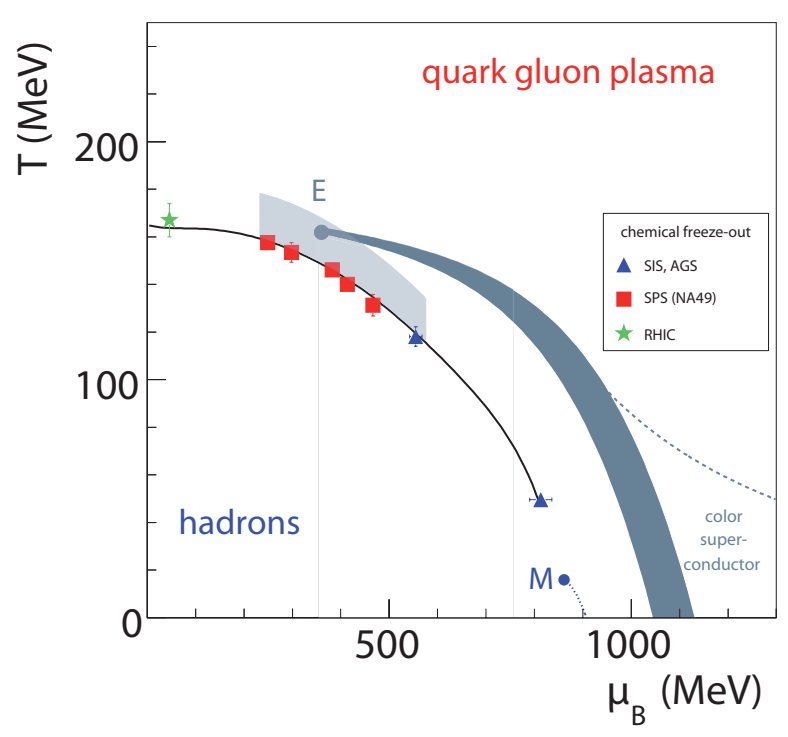

Figure 11: QCD matter phase diagram illustrating hadronic freeze-out points $[11,12,14,36,43]$ and the parton-hadron phase boundary, also indicating the expected critical point [27].

fit $[11,12,44,45]$. The situation is illustrated in Fig.11, in the $\left[\mu_{B}, T\right]$ plane. The GC freezeout points are seen to drop well below the phase coexistence line conjectured by lattice QCD [27] which, furthermore, predicts a critical point of QCD to occur in the vicinity of $\mu_{B}=300 \mathrm{MeV}$. This leads to important conclusions, firstly that the existence and location of a critical point (and thus a first order phase transition region toward higher $\mu_{B}$ ) may create critical fluctuations of the hadronic composition [46] in the lower domain of SPS energies, also affecting hadronization and the resulting hadronic ratios. This would lead to deviations from standard GC behaviour [47], which are indeed observed [48].

The second implication of Fig.11 is even more directly relevant for the arguments discussed in the present article: that hadronic freeze-out occurs later than hadronization, below $\sqrt{s} \approx 10 \mathrm{GeV}$. It thus does not occur from a preceding partonic cluster phase but from a dense hadronic medium, with mean chiral fields [43] and Goldstone bosons [21] in place, leading to short relaxation times of the participating hadrons. New mechanisms for inelastic relaxation or equilibration could dominate this medium directly at the coexistence line, such as the inverse, by detailed balance, of string or cluster decays to many hadrons [22], i.e. reactions such as

$$
\text { n pions } \leftrightarrow X \leftrightarrow \Omega \bar{\Omega}
$$

with $\mathrm{n}$ up to 10 as encouraged by the high hadron density $\rho$, which accelerates such processes in proportion to $\rho^{n}$. It is thus conceivable that the system expands while maintaining chemical equilibrium until the freeze-out temperature is reached. This equilibrium can be maintained if the main relaxation time constants are smaller than the expansion time scale (for example the volume doubling time). At higher $\sqrt{s}$ the system arrives at the phase boundary, from above, while its expansion flow velocity fields are already fully developed in the preceding partonic era [17], i.e. it "races" through the phase boundary at an expansion time scale of about $2 \mathrm{fm} / \mathrm{c}$. Hadronic "classical" 
rescattering relaxation time constants are not conceivable to fall down to,or below hadron diameter (here we disagree with ref.[21]), i.e. they can not really be much smaller than $2 \mathrm{fm} / \mathrm{c}$ with the consequence that the system freezes out in the close vicinity of the phase coexistence line. However, at low $\sqrt{s}$ there does not necessarily occur an extended partonic era (or non at all). The system reaches something like a classical turning point [46] between compression and re-expansion, where dynamical time scales are rather long. This turning point may, in fact, closely coincidence with the conjectured critical point, at low SPS energies (i.e. the system does not penetrate deeply into the partonic phase), or it may even fall below the phase boundary, all together, so that the system stays hadronic. But in either case there will occur a much slower initial re-expansion here, coinciding with maximal hadron and energy density. Thus $\tau_{\text {relax }} \ll \tau_{\text {exp }}$, and chemical equilibrium can in fact be maintained for a while, such that $T_{\text {freeze-out }}<T_{c}$. Indeed, $\tau_{\text {exp }}$ increases with falling $\sqrt{s}$, as is required by Fig.11. This indicates a new, fascinating field of experimental [48] (FAIR) and theoretical [21, 22, 43, 46, 47] progress.

\section{Conclusions}

It has been the aim of the present paper to shed a light at the apparent puzzle of hadron/resonance chemical equilibrium, observed both in elementary and in nucleus-nucleus collisions at $\sqrt{s} \geq 17$ $\mathrm{GeV}$. To this end we have first revisited the models developed in the 1970-1980s $[1,2,3,4,5]$ for hadronization as it occurs in the $e^{+} e^{-}$annihilation reaction. These models develop a QCD view that offers an evolution which ends, "naturally", i.e. without implausible ad-hoc assumptions, in a decoherent, on-shell hadron plus resonance ensemble, with a yield order which is determined by the width of available phase space (represented by an effective formation temperature) vs. the hadronic mass and spin spectrum. This situation was initially confronted by Hagedorn [8, 18] within the statistical hadronization model, and more recently by Becattini, Heinz and collaborators $[6,7,20,31]$ employing the canonical ensemble. This overall satisfactory situation in modeling elementary hadronization, which is relatively simple as no final state interaction modifies the hadronic equilibrium yield distribution (hadronization and hadronical freeze-out thus coinciding), suggests an attempt to, likewise, describe hadronization in A+A collisions. In fact, the situation can be qualitatively understood with a single additional assumption. Owing to the high spatial energy density the initial QCD DGLAP evolution branches might settle, toward the end of the non perturbative era, into extended configurations of amalgamating clusters or strings which we have called superclusters. This particular non-pertubative symbolic language may, in fact, imply a general quark gluon plasma formation process. As extended volumes should decay relatively less constrained by strict local quantum number requirements [15] but, nevertheless, under phase space governance, we thus propose that the hadronization output should now be well described by the grand canonical version of the statistical model. This implies the observed strangeness saturation systematics which is well accounted for by the model studies [11, 12, 13, 14, 15, 19, 20]. Furthermore, also the gradual transition from canonical (small volume) suppression to grand canonical saturation, with growing system size, is well documented by a wealth of experimental $[25,32,33,34,35,41]$ and theoretical $[14,15,26,36,45]$ studies.

Thus, in returning to the goal, as formulated in the introduction, we conclude that this "minimal" line of argument can, at least qualitatively, explain the overall hadronization phenomenol- 
ogy. The puzzling equilibrium distributions may well result from the stochastic and phase space influences dominating the dynamics, from late $\mathrm{pQCD}$ shower evolution to singlet cluster, or supercluster, decay to on-shell hadrons and resonances. From hadronization data alone we can thus far not derive an argument concerning the possibility that a genuine partonic equilibrium state precedes the hadonization mechanisms, at high $\sqrt{s}$ such as explored at RHIC. We note, however, that other physics observables, e.g. elliptic flow, support such a more primordial partonic equilibrium pattern [17]: an important conclusion from the RHIC data.

While insisting on the overall conclusiveness of our "minimal" line of argument (not at all a "deus ex machina" as argued in [12]) we observe, finally, that the proposals of super-rapid equilibration mechanisms, setting in within the instant of parton to hadron conversion at a high prevailing energy density $[21,22]$ in A+A collisions, need further investigation. At present they may be seen to add quantum mechanical detail (relaxation processes with time scale below hadron size can not be classical) to the overall "black box" of quantum mechanical singlet cluster/super-cluster decay to on-shell hadrons, postulated here. At lower $\sqrt{s}$, in turn, such process may be dominating the evolution toward hadronic freeze-out as we have argued in sect. 4.3.

\section{References}

[1] D. Amati and G. Veneziano, Phys. Lett. B83 (1979) 87

[2] B. R. Webber, Nucl. Phys. B238 (1984) 492

[3] J. Ellis and K. Geiger, hep-ph/9503349, and Phys. Rev. D54 (1996) 1967

[4] B. Andersson, G. Gustafson, G. Ingelman and T. Sjöstrand, Phys. Rep. 97 (1983) 33

[5] R. D. Field and S. Wolfram, Nucl. Phys. B213 (1983) 65; B. R. Webber, hep-ph/9411384; B. R. Webber, Ann. Rev. Nucl. Part. Sci. 36 (1986) 253

[6] F. Becattini, Nucl. Phys. A702 (2002) 336

[7] F. Becattini and G. Passaleva, Eur. Phys. Journ. C23 (2002) 551

[8] R. Hagedorn, CERN Yellow Report 71-72 (1971)

[9] G. Marchesini, L. Trentadue and G. Veneziano, Nucl. Phys. B181 (1980) 335

[10] T. Ericson and W. Weise, Oxford, Clarendon 1988; W. Weise, nucl-th/0504087

[11] F. Becattini, M. Gazdzicki, A. Keränen, J. Manninen and R. Stock, Phys. Rev. C69 (2004) 024905

[12] A. Andronic, P. Braun-Munzinger and J. Stachel, nucl-th/0511071, and references therein

[13] F. Becattini, J. Manninen and M. Gazdzicki, hep-ph/0511092

[14] J. Cleymans, H. Oeschler, K. Redlich and S. Wheaton, Eur. Phys. J. A29 (2006) 119; J. Cleymans, M. Stankiewicz and P. Steinberg, nucl-th/0506027; J. Cleymans, B. Kaempfer, M. Kaneta, S. Wheaton and N. Xu, Phys. Rev. C71 (2005) 054901

[15] A. Tounsi and K. Redlich, J. Phys. G28 (2002) 2095; J. Rafelski and M. Danos, Phys. Lett. B97 (1980) 279; J. Rafelski and J. Letessier, J. Phys. G28 (2002) 1819

[16] B. Müller and J. Rafelski, Phys. Rev. Lett. 48 (1982) 1066

[17] M. Gyulassy and L. McLerran, nucl-th/0405013; R. A. Lacey and A. Taranenko, nucl-ex/0610029 
[18] R. Hagedorn, Nucl. Phys. B24 (1979) 93; R. Stock, Phys. Rep. 135 (1986) 259

[19] R. Stock, Phys. Lett. B456 (1999) 277

[20] U. Heinz, Nucl. Phys. A661 (1999) 140, and talk at this conference

[21] P. Braun-Munzinger, J. Stachel and Ch. Wetterich, Phys. Lett. B596 (2004) 61

[22] C. Greiner, P. Koch-Sternheimer, F. M. Liu I. A. Shovkovy and H. Stöcker, J. Phys. G31 (2005) 725, and references therein

[23] F. Karsch, Nucl. Phys. A698 (2002) 199

[24] C. A. Salgado und U. Wiedemann, Phys. Rev. D68 (2003) 014008

[25] C. Alt et al., NA49 Coll., Phys. Rev. Lett. 94 (2005) 052301

[26] C. Hoehne, F. Puehlhofer and R. Stock, Phys. Letters B640 (2006) 96

[27] Z. Fodor and S.D. Katz, J. High Energ. Phys. 203 (2002) 14; ibidem 404 (2004) 50

[28] A. K. Wroblewski, Acta Phys. Pol B16 (1985) 379

[29] S. V. Afanasiev et al., NA49 Coll., Phys. Rev. C66 (2002) 054902, and Phys. Rev. C69 (2004) 024902

[30] S. A. Bass and A. Dumitru, Phys. Rev. C61 (2000) 064909

[31] F. Becattini and U. Heinz, Z. Phys. C76 (1997) 269

[32] D. Elia et al., NA57 Coll., J. Phys. G31 (2005) 135; F. Antinori et al., NA57 Coll., J. Phys. G32 (2006) 427; I. Kraus et al., NA49 Coll. J. Phys. G31 (2005) 147; A. Richard et al., NA49 Coll., ibidem p. 155 ; H. Caines et at al., STAR Coll., ibidem p. 101

[33] L. Ahle et al., E802 Coll., Phys. Rev. C60 (1999) 064901

[34] M. Mitrovski et al., NA49 Coll., Proceedings QM06, to be published in J. Phys. G., nucl-ex/0606004

[35] H. Caines, STAR Coll., ibidem, nucl-ex/0608008

[36] J. Cleymans, J. Phys. G28 (2002) 1575

[37] S. S. Adler et al., PHENIX Coll., Phys. Rev. C69 (2004) 034909

[38] I. G. Baerden et al., BRAHMS Coll., Phys. Rev. Lett. 90 (2003) 102301; D. Ouerdane et al., BRAHMS Coll., J. Phys. G30 (2004) 1129

[39] D. Röhrich, talk at this Conference

[40] C. Alt et al., NA49 Coll., Phys. Rev. C73 (2006) 044910

[41] G. Adams et al., STAR Coll., nucl-ex/0604014

[42] F. Becattini, private communication

[43] D. Zschiesche, talk at this Conference

[44] P. Braun-Munzinger, J. Cleymans, H. Oeschler and K. Redlich, Nucl. Phys. A697 (2002) 902

[45] J. Cleymans, talk at this Conference

[46] R. Stock, J. Phys. G30 (2004) 633

[47] V. Koch, A. Majumder and J. Randrup, nucl-th/0509030; M. Asakawa and C. Nonaka, nucl-th/050991

[48] M. Gazdzicki, talk at this Conference 